\title{
Ammonium Estimation in a Biological Wastewater Plant Using Feedforward Neural Networks
}

\author{
Hilario López García and Iván Machón González
}

Universidad de Oviedo. Escuela Politécnica Superior de Ingeniería. Departamento de Ingeniería Eléctrica, Electrónica de Computadores y Sistemas. Edificio Departamental 2. Zona Oeste. Campus de Viesques s/n. 33204 Gijón (Asturias). Spain

\begin{abstract}
Mathematical models are normally used to calculate the component concentrations in biological wastewater treatment. However, this work deals with the wastewater from a coke plant and it implies inhibition effects between components which do not permit the use of said mathematical models. Due to this, feed-forward neural networks were used to estimate the ammonium concentration in the effluent stream of the biological plant. The architecture of the neural network is based on previous works in this topic. The methodology consists in performing a group of different sizes of the hidden layer and different subsets of input variables.
\end{abstract}

\section{Introduction}

The main objective of this paper is the application of a feed-forward neural network to estimate the effluent ammonium concentration in a biological wastewater treatment plant which is composed of two serial reactors. There is a sedimentator or clarifier after each reactor. In the first reactor, there is a removal of COD, thiocyanate and phenol. On the other hand, there is an elimination of COD, ammonium and thiocyanate in the second reactor.

Although mathematical models are usually used to estimate the substrate concentrations in this type of treatment, inhibition effects between substrates can appear in certain types of wastewater. In this case, the wastewater from a coke plant of the steel industry must be treated biologically and the associated inhibition effects do not permit the use of classical mathematical models. For this reason, universal approximators such as feed-forward neural networks were used to model the biological treatment.

This work is part of the KNOWATER II project "Implementation of a Knowledge Based System for Control of Steelworks Waste Water Treatment Plant", which is sponsored by ECSC and their agreement number is 7210-PR-234. The contractors are Centro Sviluppo Materiali S.p.A., Corus RT\&D, Betrieb Forschung Institut (BFI) and Universidad de Oviedo. The main objective of the KNOWATER II project is the development of plant supervision techniques for implementation in wastewater treatment plants. 


\section{Work Undertaken}

\subsection{Process Understanding and Comprehension of the Data}

The first step is the acquisition of all the process knowledge. Technical reports, scientific articles related to the topic and expert advice must be taken into account.

The final objective is the development of an estimation of the ammonia concentration in the effluent stream of a wastewater biological treatment plant which is composed of two serial biological reactors. Taking this objective into account a feed-forward neural network was considered to estimate this concentration.

Fig. 1 to 5 represent the values of the available process variables. The measurement units are not relevant provided that the measurement has always been taken using the same unit since the data will be normalized later.

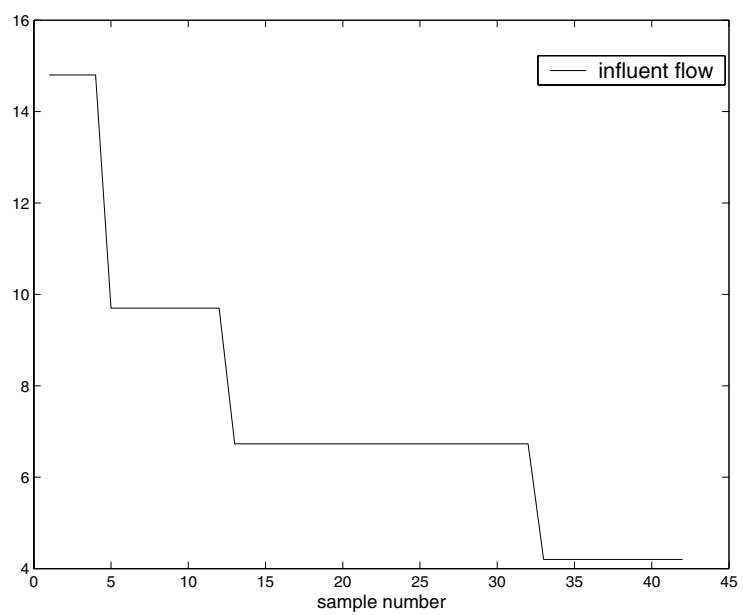

Fig. 1. Influent flow

\section{$2.2 \quad$ Selection of Variables}

One of the key tasks is the selection of the process variables to model the system. Applying the knowledge acquired during the first task mentioned above is necessary in order to obtain this selection. The physical process has to be carefully studied and the most significant process variables must be chosen. Moreover, the analysis of several combinations of process variables, which are suspected to form the training data set, will be necessary. Feeding the neural network with the significant process variables is very important.

Beforehand, the influent flow is a key variable. The higher the influent flow the lower the hydraulic residence time in a continuous reactor. Therefore the organisms might not have had enough time to reduce the concentrations of the toxic 


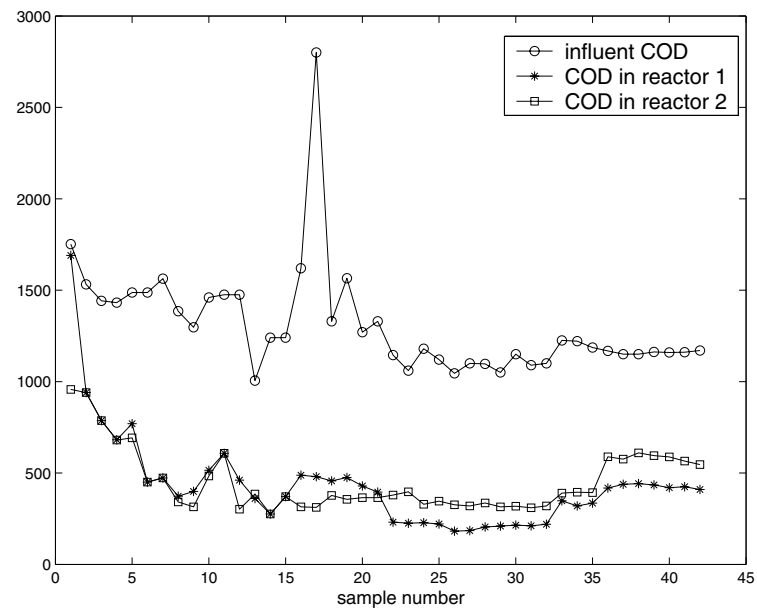

Fig. 2. Chemical oxygen demand (COD)

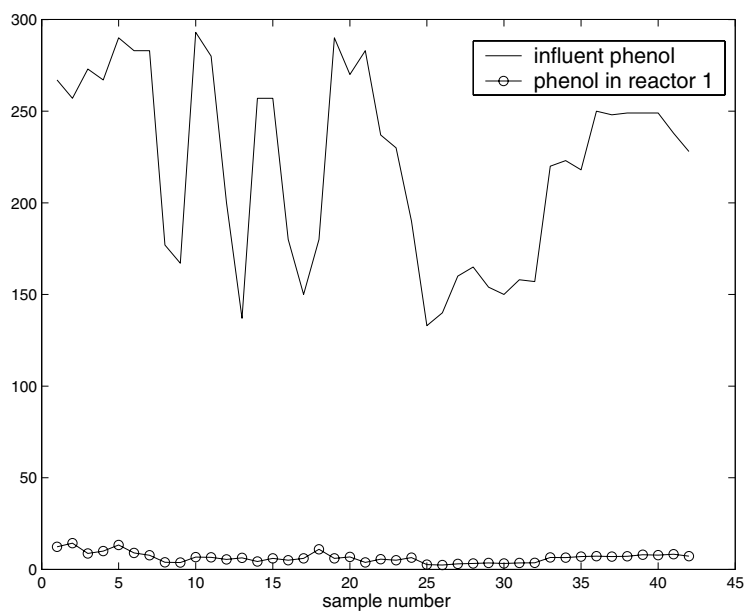

Fig. 3. Phenol concentration

substances. Thus, the influent flow and the hydraulic residence time are completely correlated and related to the reactor volume. Only one of these variables is chosen as training variable. The other would not contribute any additional information.

Fig. 1 to 5 show the available data: influent flow, organic material expressed as COD, ammonium, thiocyanate and phenol. All these variables might influence the ammonium removal. 


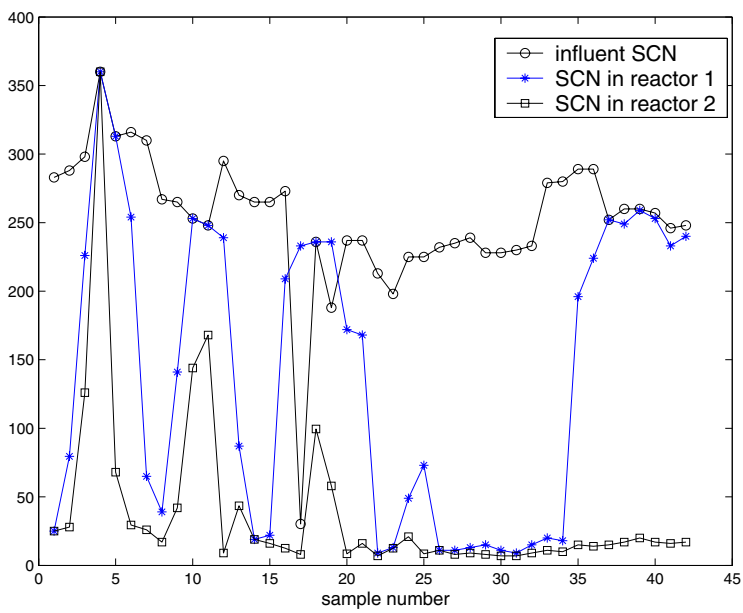

Fig. 4. Thiocyanate concentration

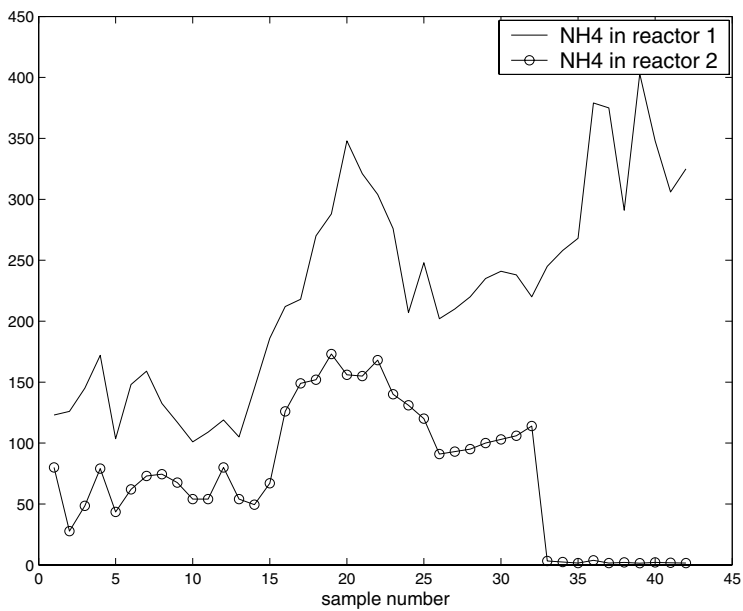

Fig. 5. Ammonium concentration

The organic material expressed as COD may influence since an increase of its concentration would demand a biological activity increment to eliminate the same rate of ammonium. For that reason, at first, this variable will be considered in two data sets.

Phenol may have the same importance given to the COD and, moreover, due to its factor of toxicity that would contribute to the treatment. However, it seems to be deduced in Fig. 3 that its influence on ammonium is minimum due to its complete removal in the first reactor whereas the ammonium is transformed into nitrate in the second reactor. For that reason the phenol concentration has not been considered to be integrated into the data set for training. 
Table 1. Training variables

\begin{tabular}{|l|l|}
\hline Set number & Process variables \\
\hline Variable set No 1 & $\begin{array}{l}\text { Influent flow, influent ammonium concentration, } \\
\text { influent thiocyanate concentration and influent COD }\end{array}$ \\
\hline Variable set No 2 & $\begin{array}{l}\text { nffluent flow, influent ammonium concentration } \\
\text { and influent COD }\end{array}$ \\
\hline Variable set No 3 & Influent flow and influent ammonium concentration \\
\hline Variable set No 4 & $\begin{array}{l}\text { Influent flow, influent ammonium concentration } \\
\text { and influent thiocyanate concentration }\end{array}$ \\
\hline
\end{tabular}

The same mentioned above is valid for the thiocyanate but its elimination happens in both reactors and is not complete. Thus, it is considered to form part of the training data set.

Finally, the influent ammonium concentration must be considered since it obviously influences the effluent ammonium concentration.

Taking the above mentioned into account, four variable sets will be analyzed as input variables which are described in table 1. Several models of neural networks will be trained using these four variable sets to select the best model that minimizes the estimation error.

\subsection{Data Preprocessing and Training}

The data are normalized to a zero mean and a unitary variance. This allows all the features to move in the same ranges and, hence, be treated by the neural network in the same way.

The next step consists in establishing the architecture of the neural network which is composed of a single hidden layer with hyperbolic tangent as activation function and a single neuron with linear activation function as output layer, see Fig. 6.

The activation function of the neurons of the hidden layer is a hyperbolic tangent. In this way, this type of function allows the network to learn non linear

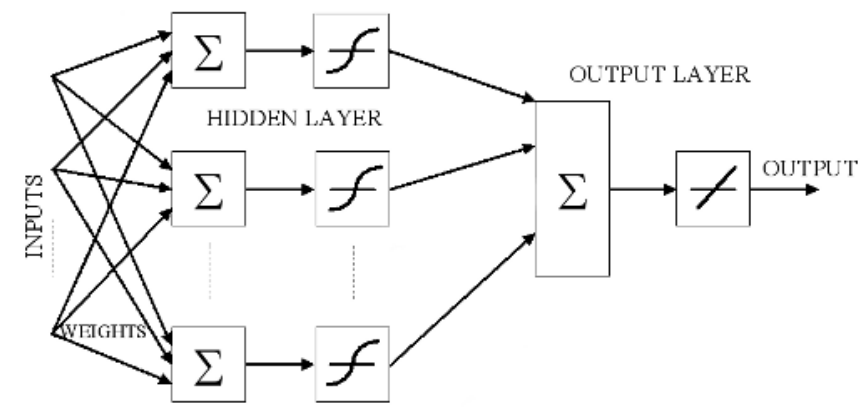

Fig. 6. Architecture of the neural network 
relationships. The activation function of the output layer is linear enabling the network to take any value.

This network topology can be used as a general approximator for any function that has a finite number of discontinuities whenever the hidden layer has a sufficient number of neurons and non linear activation function, see [1], 2], 3] and [4].

The training of the neural network was carried out using the LevenbergMarquardt algorithm [5], 6] and [7].

After training, the network is pruned removing the weights that have the lowest saliences [8]. At this stage a data set different from that used for training is employed. This utilized data set is the testing data set.

\section{Methodolgy of Model Validation}

The prediction of the model must be as accurate as possible for all the process working zones. The model validation to achieve this requirement attempts to select the best model according to the mean square error (mse). The mse for different assays (both training and pruning) were carried out. In this work, 60 assays were done for each variable data set and different number of hidden neurons. The minimum value, the mean value and the standard deviation of the mse of these 60 assays are registered. The mean square error is calculated as (1)

$$
e_{t}=\frac{1}{N} \sum_{i=1}^{N}\left(\hat{y}_{i}-y_{i}\right)^{2}
$$

where $y$ is the real value, $\hat{y}$ is the estimated value, $e$ is the estimation error. These three values are calculated for each sample $i . N$ is the total number of samples. The autocorrelation of the prediction error is useful as well [9].

The objectives of this validation process are:

1. Discover the minimum number of input variables that yield the best estimation of the objective variable (effluent ammonium concentration).

2. The architecture of the neural network: Number of layers, number of neurons in each layer and activation functions.

Discovering the input variables that optimize the approximation to the objective function is the first task based on the topology described above. The mean square error and the autocorrelation between the output variable and the error are useful to carry out this task. Once the best combination of input variables has been selected, the number of hidden neurons must be determined. A low number of neurons does not provide enough parameters to train the neuronal network correctly. On the other hand, an excessive number of neurons leads to overtraining problems and its computational cost is higher. 


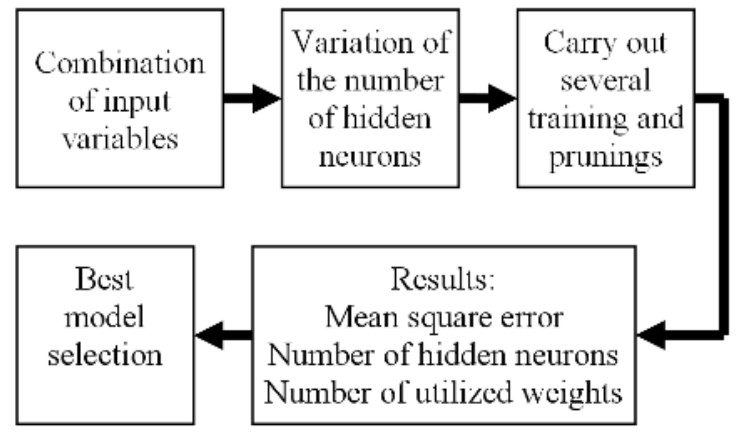

Fig. 7. Methodology for training and validation

The training and pruning of the neuronal network has been carried out using the toolbox named "Neural Based Network Identification System" developed by the Technical University Helsinki [10] and [11.

Fig. 7 represents the methodology. The number of neurons in the hidden layer is increased gradually for each combination of input variables, making several tests with each of them (60 assays were carried out in this work). After pruning, the results are registered as the number of neurons in the hidden layer, the testing and training errors, the average and variance of these errors and the final number of utilized weights.

\section{Results}

Fig. 8 shows the minimum value, mean value and standard deviation of the mean square error in function of the number of utilized weights calculated for each combination of variables of table 1 .

It can be seen that the higher the number of weights the lower the training error whereas at the beginning the testing error decreases, although it rises later. Therefore an optimum number of weights must be found.

Variable set No 4 or the influent flow, the influent ammonium concentration and the influent thiocyanate concentration have been chosen as input variables based on the minimum value, the mean value and the standard deviation of the testing mean square error. The best results take place in a number of utilized weights equal to 18 which correspond to an original model of 8 hidden neurons. Problems of local minima have been detected in some models.

An iterative loop is established to search for the best model that minimizes the testing mean square error after pruning and considering the selected input variables and a number of hidden neurons equal to 8. Fig. 9 represents the best model after pruning. Four of the eight neurons are not used as can be observed. 

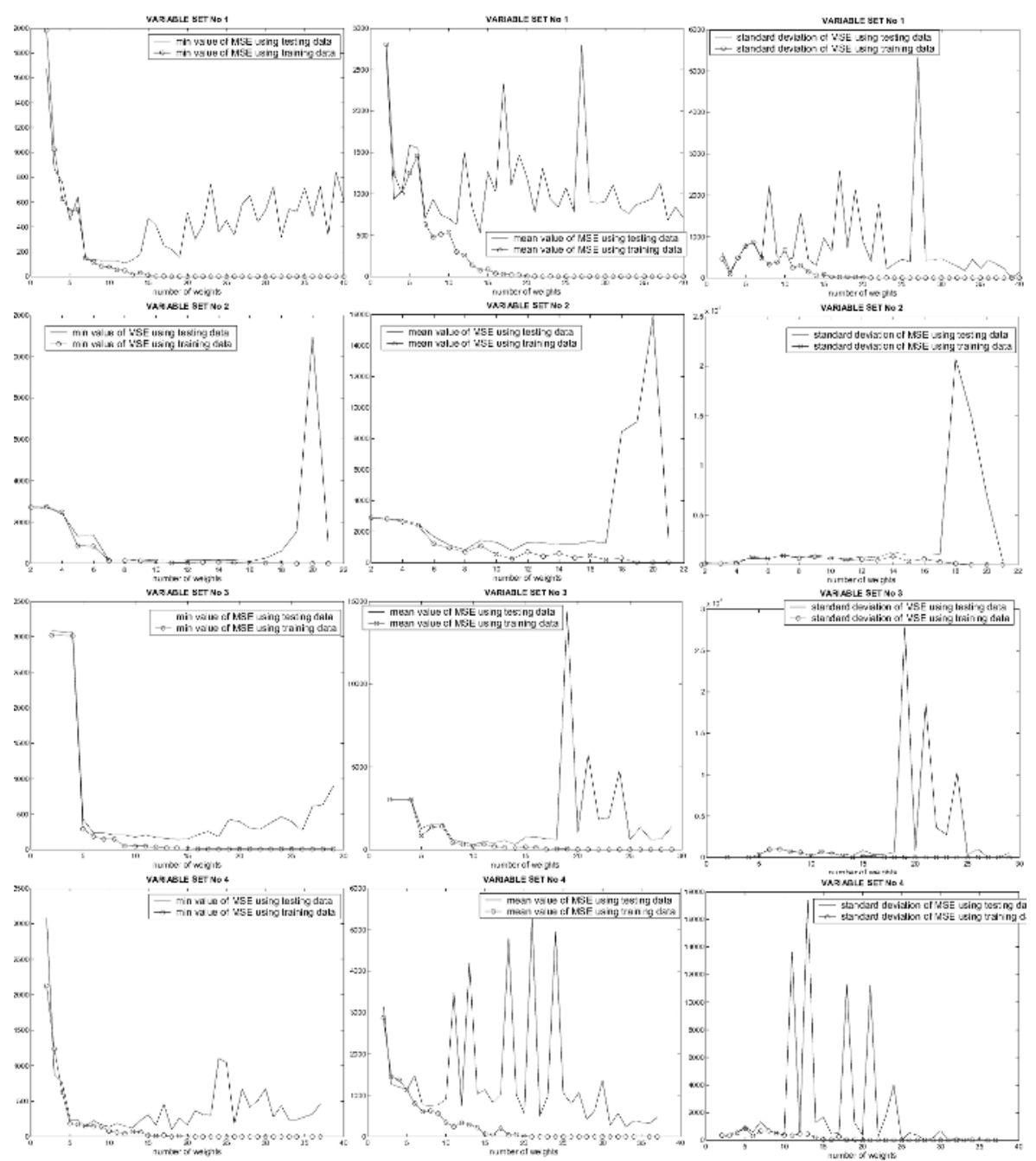

Fig. 8. Minimum value, mean value and standard deviation of the mse in function of the number of utilized weights for each variable set

However, it is necessary to start training with a high enough number of neurons and then stop the procedure and remove the pruned weights.

Fig. 10 shows the real data, both training and testing data, corresponding to effluent ammonium concentration and the values estimated by the neural network. The autocorrelation of the estimation error is good tending quickly to zero and the distribution is also good with most of the samples centered in the origin according to Fig. 11 . 


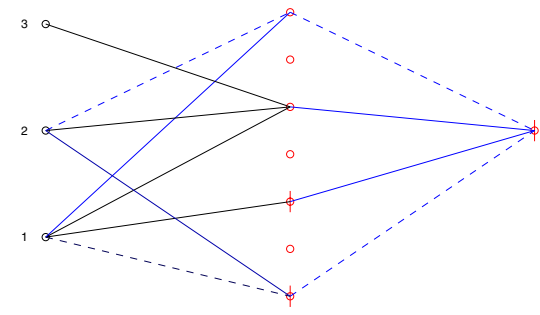

Fig. 9. Best of model of the neural network
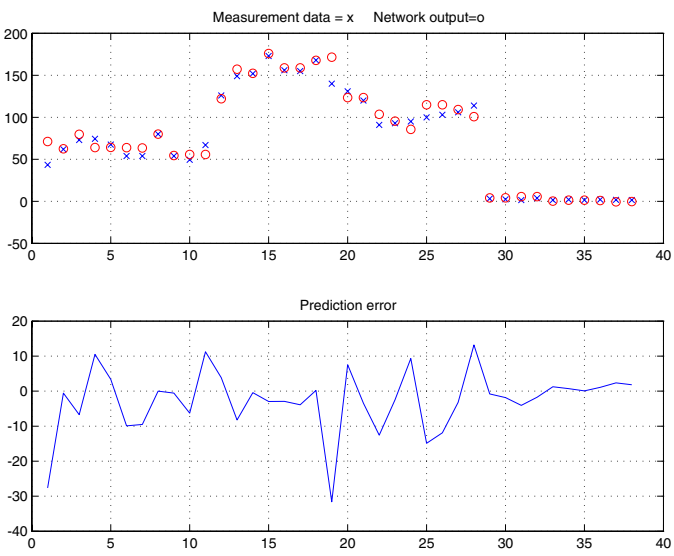

Fig. 10. Estimated and real effluent ammonium concentration
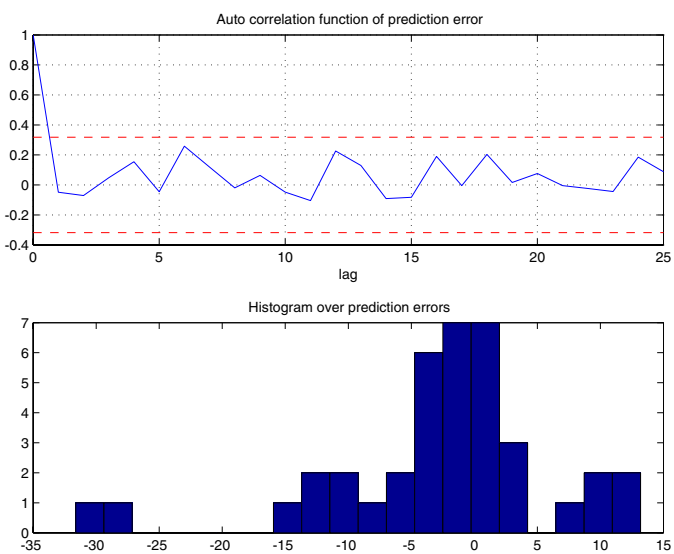

Fig. 11. Autocorrelation of the estimated error 


\section{Conclusions}

A neural network model has been developed to estimate the ammonium concentration in the effluent stream of a wastewater plant whose treatment is biological. There are mathematical models well known for biological treatment such as the Activated Sludge Model (ASM). These can be formulated using kinetic dynamics (Haldane, Monod) and material balances to configure the particular structure of the plant. However, in this case these models are not very useful because of the existence of inhibition between substances. In this sense, the use of ANNs has been recommended. Neural networks are widely used to estimate key parameters of physical processes.

In this paper, a feed-forward neural network is outlined to obtain a satisfactory approach to estimate the effluent ammonium concentration of the treatment plant. The methodology consists in performing a group of different sizes of the hidden layer and different subsets of input variables.

The developed model is useful to obtain simulations under varying conditions of the influent stream. In this way, the effluent ammonium concentration can be estimated. This neural network achieves better results than the classical mathematical models for biological wastewater treatment due to the problematic composition of the coke wastewater.

\section{Acknowledgments}

Our warmest thanks are expressed to the following for their financial support: The Commission of the European Communities, European Coal and Steel (ECSC), supporting KNOWATER II project "Implementation of a Knowledge Based System for Control of Steelworks Waste Water Treatment Plant" with 7210-PR-234 as agreement number.

\section{References}

1. Hornik, K., Stinchcombe, M., White, H.: Multilayer feedforward networks are universal approximators. Neural Networks. 2 (1989) 359-366

2. Funahashi, K.: On the approximate realization of continuous mappings by neural networks. Neural Networks. 2 (1989) 192-193

3. Cybenko, G.: Approximation by superpositions of a sigmoidal function. Mathematics of Control, Signals, and Systems. 2 (1989) 303-314

4. Hartman, E., Keeler, J., Kowalski, J.: Layered neural networks with gaussian hidden units as universal approximations. Neural Computation 2 (1990) 210-215

5. Levenberg, K.: A method for the solution of certain problems in least squares. Quart. Appl. Math. (1944) 164-168

6. Marquardt, D.: An algorithm for least-squares estimation of nonlinear parameters. SIAM J. Appl. Math. 11 (1963) 431-441

7. Moré, J.: The levenberg-marquardt algorithm: Implementation and theory. Numerical Analysis. 630 (1977) 105-116 
8. LeCun, Y., Denker, J., Solla, S. Optimal brain damage. Advances in Neural Information Processing Systems. 2 (1990) 598-605

9. López, H., González, R., Machón, I., Ojea, G., Peregrina, S., González, J., de Abajo, N.: Identification of melting and brightening section of a tinplate facility by means of neural networks. Proc. European Control Conference. Porto, (2001)

10. Norgaard, M., Ravn, O., Poulsen, N., Hansen, L.: Neural Networks for Modelling and Control of Dynamic Systems. London: Springer-Verlag (2000)

11. Norgaard, M., Ravn, O., Poulsen, N.: Nnsysid-toolbox for system identification with neural networks. Mathematical and Computer Modelling of Dynamical Systems. 8 (2002) 1-20 